\title{
Meiotic and mitotic instability of two EMS-produced centric fragments in the haplodiploid wasp Nasonia vitripennis
}

\author{
MARIE-JEANNE PERROT-MINNOT* $+\$$ \& JOHN H. WERREN\$ \\ $\dagger$ Équipe Écologie Évolutive, UMR-CNRS 5561 Biogéosciences, Université de Bourgogne, 6 boulevard Gabriel, \\ 21000 Dijon, France and $\ddagger$ Biology Department, University of Rochester, Rochester, NY 14627, U.S.A.
}

\begin{abstract}
Terminal deletions that result in chromosomal fragments with centromeres (centric fragments) are relatively easy to generate and study in the haplodiploid insect Nasonia. We investigated the transmission stability of two chromosomal fragments generated by chemical mutagenesis. Visible mutations at the $R$ locus (peach-233 and St-DR) and a linked body-colour mutant (purple) were used to track transmission of the centric fragments (which lack the purple locus and are wild-type at the $R$ locus). Transmission rates in meiotic oogenesis were low (medians 0.15-0.18) and comparable to previous data on centric fragments in this species. The homologous chromosome genetic background strongly affected meiotic stability of one centric fragment (CF2) but not the other (CF1). Specifically, in peach/scarlet $R$ locus heterozygous females, CF2 showed a normal segregation proportion with the chromosome bearing the scarlet allele (0.16), but near complete failure to segregate with peach (0.0002). Data show that this is due to loss of CF2 in eggs receiving peach, rather than to preferential segregation of CF2 with scarlet or mortality of CF2-bearing males. CF1 shows typical segregation ratios with both chromosomes. We hypothesize that deletions (or rearrangements) associated with the peach-233 mutant inhibit proper pairing and segregation of CF2. Consistent with the model, CF2 did segregate with chromosomes that had undergone recombination between peach and purple (a bodycolour mutation $10 \mathrm{~cm}$ from peach), indicating that the domains inhibiting segregation are closely linked to peach. Mitotic instability also differed between the two fragments; reduced mitotic stability may relate to absence of telomeres on these centric fragments. Given the relative ease of generating and tracking terminal deletions in Nasonia, we propose this as a good system for studying mitotic and meiotic stability of centric fragments. Finally, results are discussed in relation to the evolution of B chromosomes from centric fragments.
\end{abstract}

Keywords: B-chromosome, centric fragment, EMS, haplodiploid, mitotic instability, mutagen.

\section{Introduction}

A variety of chemical and environmental mutagens result in chromosomal deletions and rearrangements (Sankaranarayanan, 1996; Grant \& Owens, 1998). Terminal deletions are an extreme form; one or both arms of a chromosome are deleted, leaving the centromeric region and flanking regions of variable size. Such largescale deletions are typically lethal or highly detrimental, because they are often associated with aneuploidy or dosage compensation effects, including partial trisomy of pericentric regions in humans (Stankiewicz et al., 2000). Under some circumstances, major terminal

*Correspondence. E-mail: mjperrot@u-bourgogne.fr deletions can result in a chromosome containing only the centromere and a relatively small flanking region. These are referred to as 'centric fragments'. In humans, they are referred to as 'marker chromosomes' and are implicated in the aetiology of abnormal phenotypes (Li et al., 2000; Stankiewicz et al., 2000). Terminal deletions also typically lack telomeres on the deleted arm, which can result in chromosome instability due to fusion-bridge-breakage cycles at the unprotected ends of the chromosome (McClintock, 1942; Zakian, 1995). In some cancer cells, chromosomal instability has been shown to produce marker chromosomes (Pai et al., 1999).

In contrast to many other organisms, such as Drosophila (Ahmad \& Golic, 1998), centric fragments 
are easily recovered in the haplodiploid insect, Nasonia vitripennis. Whereas in most organisms, high levels of paternal chromosome fragmentation result in embryonic lethality, in Nasonia it can result in haploidization of the embryo, allowing normal development of the embryo into a male. This permits recovery of centric fragments that have 'survived', so long as they do not cause lethality due to dosage compensation problems (diploidy at some loci). In addition, several visible markers are available that occur near centromeric regions (Ryan et al., 1985; Gadau et al., 1999), allowing easy screening for and tracking of centric fragments carrying wild-type alleles at those loci. Therefore, the study of meiotic and mitotic instability of terminal deletions and (specifically) centric fragments is possible.

The aim of the present study was to analyse the transmission patterns of two centric fragments $(\mathrm{CF})$ in the parasitoid wasp Nasonia vitripennis. Chromosomal fragments can be experimentally generated in Nasonia by irradiation, chemical mutagens, Wolbachia-induced cytoplasmic incompatibility or interspecific hybridization (Ryan et al., 1985, 1987; Beukeboom \& Werren, 1993b; Perfectti \& Werren, 2001). We generated CFs by the chemical mutagen ethyl methanesulfonate (EMS). Three factors of differential transmission rates and instability were considered: the type of gametogenesis - meiotic or mitotic, the genetic composition of the autosomal region homologous to the centric fragment, and intrinsic differences between the fragments. Arrhenotoky in this species allows for a comparison of $\mathrm{CF}$ stability in meiosis and in mitosis in the same nuclear and cytoplasmic backgrounds. Spermatogenesis in haploid males is mitotic whereas oogenesis in diploid females is meiotic. We used phenotypic mutants in the corresponding autosomal region on the centric fragments to estimate $\mathrm{CF}$ stability in association with different genotypes. The recessive mutations used affect eye-colour and body-colour, two loci belonging to the linkage group I of $N$. vitripennis (Saul, 1989). The interest in the eye-colour mutations lies in their location in the complex $R$ locus, comprising a group of four non-recombining sites governing eye-colour (Saul, 1989). This ' $R$-complex' appears to be close to the centromere, based on previous centric fragment studies (Ryan et al., 1985) and molecular marker maps (Gadau et al., 1999). Our data confirm the close location of the $R$-complex to a centromeric region. They also reveal a site involved in pairing or stable association between the centric fragment and its homologous autosome in meiosis. The relevance of our observations on centric fragment instability to chromosome pairing and B-chromosome evolution is discussed.

\section{Materials and methods}

\section{Strains used}

Three strains were used in the experiment. Two strains (peach-purple and st-DR) include genes within the $R$-complex. The $R$-complex is a region of no recombination composed of several genes involved in eye-colour and viability. The $R$-complex contains four loci affecting eye-colour (labelled $O, S, M, P$ ), based upon complementation tests. Several lines of evidence suggest that this region occurs close to the centromere (Ryan et al., 1985; Gadau et al., 1999), which may explain the lack of recombination within the region.

St-DR $(s t): s t-D R$ is a recessive $R$-complex eye-colour mutation causing scarlet eyes. Among the four loci governing eye-colour at the $R$-complex, the $s t-D R$ (hereafter referred to as $s t$ ) corresponds to a mutation at the $S$ locus $(+s t+++$ ), based upon complementation studies (Ryan et al., 1985).

Peach-purple (pepu): the strain Peach-purple carries the recessive eye-colour mutation peach and the bodycolour mutation purple. Peach-233 (hereafter referred to as $p e$ ) is an $R$-complex mutation that is a double mutation at the $O$ and $S$ sites (pe st ++ ) (Ryan et al., 1985). Therefore, pe possibly involves a deletion encompassing these two loci. There is no recombination between st and pe, and heterozygous st/pe females are phenotypically scarlet. Purple is a recessive body-colour mutation that maps approximately $10 \mathrm{cM}$ distally to the $R$-complex (linkage group I) (Saul et al., 1965).

Paternal-sex-ratio (PSR): PSR is a supernumerary chromosome that is transmitted through sperm but causes elimination of paternal chromosomes following fertilization (Werren, 1991); all-male (haploid) families result. The PSR strain is in a wild-type background with respect to $R$-complex loci and the purple body locus.

\section{Production of centric fragments}

The chromosomal fragments were generated using ethyl methanesulfonate (EMS) as part of studies attempting to generate mutations in the paternal-sex-ratio (PSR) chromosome of $N$. vitripennis (Werren, 1991). For mutagenesis, adult males from a wild-type strain carrying the PSR chromosome were fed a $10 \%$ sucrose solution containing $50 \mathrm{~mm}$ EMS for $7 \mathrm{~h}$ (Grigliatti, 1986). Treated males were crossed to scarlet-eyed females $(s t / s t)$. Some males in such crosses do not carry the PSR chromosome and therefore typically produce female progeny. However, in a few crosses, individual male progeny from such fathers showed mosaic eye colour (wild-type and scarlet). Previous studies indicate 
that such mosaicism is typical of males with centric fragments retaining the wild-type locus for eye-colour (Ryan et al., 1985, 1987). Three mosaic males were rescued, harbouring a fragment hereafter referred to as $\mathrm{CF} 1, \mathrm{CF} 2$ or $\mathrm{CF} 3$. The third fragment was lost at the next generation, possibly due to a very high instability. The transmission patterns of the two others $(\mathrm{CF} 1$ and $\mathrm{CF} 2)$ are presented here. Both $\mathrm{CF} 1$ and $\mathrm{CF} 2$ include wild-type alleles for the $R$-complex; in fact, that is how they were detected (production of wild-type haploid males from $s t / s t$ females). The presence of wild-type alleles in the $R$-complex allows tracking of the centric fragments and investigations of their transmission dynamics.

\section{Cytology}

The presence of a chromosomal fragment was confirmed by cytological studies on squashes of third-instar brain tissues stained with lacto-aceto-orcein, using a 'squasheasy' method (Nur et al., 1988.). Basically, the brain of a pupa at the red-eye stage was dissected out and kept in a solution containing $0.8 \% \mathrm{KCl}$ and $0.2 \%$ colchicine for $1 / 2 \mathrm{~h}$. The brain was then rinsed in $70 \%$ ethanol, fixed in a $4: 1$ mixture of $95 \%$ ethanol and glacial acetic acid, and transferred to a drop of orcein stain $(2 \%$ in $50 \mathrm{~mL}$ glacial acetic acid, $30 \mathrm{~mL}$ lactic acid and $20 \mathrm{~mL} \mathrm{H}_{2} \mathrm{O}$ ) on a coverslip. A clean slide was lowered over the drop of stain, and then inverted to pick up the coverslip. Cells were spread by squashing with increasing pressure. Preparations were viewed under a light microscope at $15 \times 40$ magnification.

\section{Crosses}

We conducted crosses to estimate transmission rates of the two fragments via males (mitosis) and females (meiosis), and for various genotypes at the homologous region, including the eye-colour $R$ locus. The pepu and st-DR strains were used. As mentioned above, the pe and st mutations are recessive and appear as wildtype eye colour either in heterozygous females $(s t /+$ or $p e /+$ ), or in homozygous recessive females and hemizygous recessive males carrying the fragment. The st allele is dominant over pe: the genotype of scarlet females is either st/st or st/pe. For simplicity of reading, the centric fragment associated with a genotype is labelled $\mathrm{fr}^{+}$, the two-locus genotype is given in italics for haploids $s t+$ and diploids $s t+/ s t+$, and the eyecolour and body-colour phenotype is indicated in quotation marks 'st + '. The two mosaic males rescued with $\mathrm{fr}^{+}$will be referred to as $\mathrm{F}_{0}$ males. Crosses were performed over three generations to test for differential stability of the fragments in mitosis and meiosis, in association with $s t+$, pepu, and recombinant stpu and pe + genotypes.

Transmission rates were estimated for both fragments in $\mathrm{F}_{0} s t+f r^{+}$males and in $\mathrm{F}_{1}$ homozygous $s t+$ $/ s t+f r^{+}$and heterozygous $s t+/$ pepu $f r^{+}$females. Further observations were done on CF2 instability in $F_{2}$ $s t+\mathrm{fr}^{+}$and stpu $\mathrm{fr}^{+}$males and in $\mathrm{F}_{3}$ stpu/pepu $\mathrm{fr}^{+}$ females. Virgin males and females of known genotype were individually paired upon emergence. After mating, females were set on two fleshfly pupae (Sarcophaga bullata) for oviposition. Alternatively, females carrying the fragment could be set on the host as virgins, to score transmission rate to male (haploid) offspring. The offspring phenotypes were scored on live $\mathrm{CO}_{2^{-}}$ anaesthetized adult wasps based upon adult eye and body-colour. Transmission rate data were analysed with non-parametric statistics (Mann-Whitney $U$-test and Wilcoxon paired test) (LOGITHEQ software).

\section{Assays for paternal (mitotic) and maternal (meiotic) transmission}

Because of the difference in male (mitotic) and female (meiotic) gametogenesis in haplodiploids, we can indirectly assay for meiotic vs. mitotic transmission rates of centric fragments. Males carrying the fragment were identified by (at least some) wild-type eye colour in a mutant genetic background at the $R$-complex (st or pe). Transmission of the centric fragment from these males was detected if their daughters had wild-type eyes in a mutant genetic background (st/st or st/pe, both of which without the fragment would give scarlet eyes). Because males are haploid and have haploid non-reductive gametogenesis, incomplete transmission of the centric fragment is a measure of its mitotic instability. No meiotic divisions were involved. However, it's transmission is a composite of the mitotic instability through male germ line cell lineages to sperm, then to the fertilized egg (female), and then to somatic cell lineages leading to cells in the female's eyes.

Similarly, maternal (meiotic) instability was measured by selecting females with wild-type eyes in a mutant background and scoring for wild-type eye colour among their male (or female) progeny with a mutant genotypic background. This is a composite of transmission through female oogonial cell lineages, through meiosis to the egg, followed by somatic cell lineages leading to cells in the offspring's eyes. The differences involve male vs. female germ cell lineages, and most importantly, a meiotic cell division in maternal gametogenesis vs. a mitotic cell division in the paternal gametogenesis. However, in both cases, males or females mosaic for the centric fragment but with it absent in their eyes tissues would be scored as fragment-negative.

(C) The Genetics Society of Great Britain, Heredity, 87, 8-16. 


\section{Results}

The comparison of transmission rates of $\mathrm{CF} 1$ and $\mathrm{CF} 2$ at $F_{0}$ and $F_{1}$ generations revealed large differences in stability (Tables 1 and 2). Their transmission patterns are therefore presented separately after some common features are described. The transmission of CF1 and $\mathrm{CF} 2$ from heterozygous $s t+/ p e p u f r^{+} \mathrm{F}_{1}$ virgin females carrying the $p u$ mutant allele at the body-colour locus produced purple males with wild-type eye colour (due to $+R$-complex alleles carried by the fragment) (Table 2). Therefore, CF1 and CF2 did not include the bodycolour locus, and the terminal deletions apparently end somewhere within the $10 \mathrm{cM}$ region between the $R$-complex and $p u$. Since wild-type males at the eyecolour locus were produced by both peach $\mathrm{fr}^{+}$and scarlet $f r^{+}$males, both fragments included at least the $O$ and $S$ sites of the $R$-complex. These two fragments were therefore comparable to the $O^{+} S^{+}$fragments generated in unidirectionally incompatible crosses by Ryan et al. (1985).

\section{Cytology}

Cytological examinations revealed the presence of a fragment clearly identifiable in haploid and diploid cells of young pupae (Fig. 1). In polar view of haploid metaphase cells, the fragment was usually lying in the centre of the configuration (Fig. 1A-C), as already observed by Ryan et al. (1985). In some diploid cells, a possible homologous autosome was found with the fragment in the centre of the metaphase plate with the nine chromosomes laid out as a ring (Fig. 1D, 1E).

\section{Transmission pattern of CF1}

The CF1 fragment was fairly unstable in male gametogenesis, based on a low transmission through male (mitotic) spermatogenesis $(0.38$, Table 1$)$. The transmission rate was lower than 0.5 in all of the nine families analysed, i.e. much lower than the expected transmission ratio in mitosis (one). It is therefore possible that $\mathrm{CF} 1$ did not always segregate properly to daughter cells in mitosis, at least within the cell lineages leading to spermatogonia. However, we cannot rule out that the instability of these chromosomes occurred following spermatogenesis and fertilization, in the early developing embryo of the male's progeny. Appearance of eye-colour mosaicism suggests that instability also occurred in later stages of somatic cell divisions, and was not restricted to early divisions. Mitotic instability could be due in part to fusion-bridge-breakage cycles caused by lack of telomeres on the centric fragment (McClintock, 1942). Mitotic pairing could also constrain the migration of the centric fragment to one of the two daughter cells.

The transmission rate from $F_{1}$ virgin females to their haploid sons (including meiotic transmission) was low, with only $15 \%$ to $18 \%$ of male progeny inheriting the fragment. The lower transmission rates of $\mathrm{CF} 1$ in meiosis $\left(\mathrm{F}_{1} s t+/ s t+f r^{+}\right.$females) compared to mitosis $\left(\mathrm{F}_{0} s t+\mathrm{fr}^{+}\right.$males) in the same genetic background (scarlet) is statistically significant (Mann-Whitney test, $\left.U=0, n_{1}=9, n_{2}=59, P<0.001\right)$. This pattern is similar to that observed with other centric fragments (Ryan et al., 1985; Beukeboom \& Werren, 1993b), and reflects segregational loss of the fragment in meiosis (see below). A slightly but significantly higher transmission rate was observed in heterozygous $s t+/ p e p u$ compared to homozygous $s t+/ s t+$ females (Mann-Whitney test, $U=1201.5, n_{1}=61, n_{2}=59, P<0.01$ ) (Table 2). No difference was found in the transmission rate of CF1 to $p u$ vs. + males, in contrast to results for CF2 (below).

\section{Transmission pattern of CF2}

CF2 shows markedly different transmission patterns. Mitotic transmission rates of CF2 were higher than for

Table 1 Transmission rate of CF1 and CF2 centric fragments via males in Nasonia vitripennis. $\mathrm{F}_{0}$ males were mated to $s t+/$ $s t+f r^{-}$or pepu/pepu $f r^{-}$females and $\mathrm{F}_{2}$ males were mated to $s t+/ s t+f r^{-}$females. In all crosses, the phenotype of $\mathrm{F}_{1}$ and $\mathrm{F}_{3}$ female offspring carrying $f r^{+}$was ' $++^{\prime}: f r^{+}$transmission is the ratio of the number of ' ++ ' females to total number of females produced in a single cross

\begin{tabular}{|c|c|c|c|c|c|c|c|}
\hline \multirow{2}{*}{$\begin{array}{l}\text { Centric } \\
\text { fragment }\end{array}$} & & \multicolumn{2}{|c|}{ Male } & \multirow[b]{2}{*}{$n$} & \multirow{2}{*}{$\begin{array}{l}\text { Mean family } \\
\text { size (SD) }\end{array}$} & \multirow{2}{*}{$\begin{array}{l}\text { Total no. of } \\
\text { female progeny }\end{array}$} & \multirow{2}{*}{$\begin{array}{c}\text { Median } f^{+} \\
\text {transmission } \\
(\text { min-max })\end{array}$} \\
\hline & & Phenotype & Genotype $\dagger$ & & & & \\
\hline $\mathrm{CF} 1$ & $\mathrm{~F}_{0}$ & '+ +' & $s t+f r^{+}$ & 9 & $205(55.5) \sharp$ & 1624 & $0.38(0.28-0.45)$ \\
\hline $\mathrm{CF} 2$ & $\mathrm{~F}_{0}$ & $'++$ & $s t+f r^{+}$ & 10 & $209.6(38.5) \ddagger$ & 1761 & $0.76(0.63-0.84)$ \\
\hline $\mathrm{CF} 2$ & $\mathrm{~F}_{2}$ & $'++$ & $s t+($ or $p e+) f r^{+}$ & 30 & $48.1(14.2)$ & 1086 & $0.82(0.59-0.94)$ \\
\hline $\mathrm{CF} 2$ & $\mathrm{~F}_{2}$ & $'+p u '$ & stpu (or pepu) $f r^{+}$ & 14 & $87.7(16)$ & 975 & $0.73(0-0.91)$ \\
\hline
\end{tabular}

$\dagger F_{2}$ males: see text for the interpretation of $F_{2}$ male genotypes (the less frequent genotype is shown in parentheses).

\#Family size in crosses with $\mathrm{F}_{0}$ males are higher because females were re-hosted twice (six hosts overall instead of two).

(c) The Genetics Society of Great Britain, Heredity, 87, 8-16. 
Table 2 Transmission rates of two centric fragments $f r^{+}(\mathrm{CF} 1$ and $\mathrm{CF} 2)$ in $\mathrm{F}_{1}$ virgin females of Nasonia vitripennis

\begin{tabular}{|c|c|c|c|c|}
\hline \multirow{2}{*}{$\begin{array}{l}\text { Genotype of } F_{1} \text { virgin females } \\
\text { Centric fragment }\end{array}$} & \multicolumn{2}{|c|}{$s t+/ p e p u f r^{+}$} & \multicolumn{2}{|c|}{$s t+/ s t+f r^{+}$} \\
\hline & CF1 & $\mathrm{CF} 2$ & $\mathrm{CF} 1$ & $\mathrm{CF} 2$ \\
\hline Number of crosses, $n$ & 61 & 61 & 59 & 50 \\
\hline Male phenotypes & & & & \\
\hline 'st + ' & 2303 & 2544 & 3916 & 4662 \\
\hline 'pe pu' & 2325 & 3092 & - & - \\
\hline 'st $p u$ ' (rec.) & 181 & 141 & - & - \\
\hline 'pe+' (rec.) & 162 & 166 & - & - \\
\hline${ }^{\prime}++^{\prime} \mathrm{fr}^{+}$ & 622 & 491 & 680 & 902 \\
\hline$'+$ pu' $f r^{+}$ & 574 & 27 & - & - \\
\hline Total number produced & 6169 & 6462 & 4596 & 5564 \\
\hline Mean family size (SD) & $101.1(25.7)$ & $105.9(23.6)$ & $94.3(21.8)$ & $93.8(22.2)$ \\
\hline Recombination rate & $0.06(0.04-0.09)$ & $0.05(0.04-0.06)$ & - & - \\
\hline Proportion 'pe' without $f r^{+} \dagger$ & $0.51(0.46-0.53)$ & $0.56(0.51-0.60)$ & & \\
\hline Transmission rate over all & $0.18(0.15-0.24)$ & $0.08(0.06-0.1)$ & $0.15(0.13-0.19)$ & $0.15(0.12-0.18)$ \\
\hline Proportion ' + +'/fs & $0.10(0.08-0.12)$ & $0.08(0.05-0.1)$ & - & - \\
\hline Proportion '+ ${ }^{\prime} u^{\prime} / \mathrm{fst}$ & $0.09(0.06-0.12)$ & $0(0-0.01)$ & & \\
\hline $\begin{array}{l}\text { Proportion ' }++ \text { ' among wild- } \\
\text { type body-colour males }\end{array}$ & $0.20(0.15-0.24)$ & $0.16(0.11-0.19)$ & & \\
\hline $\begin{array}{l}\text { Proportion ' }+p u \text { ' among } \\
\text { purple males }\end{array}$ & $0.17(0.13-0.23)$ & $0(0-0.02)$ & & \\
\hline
\end{tabular}

The number of individuals in each phenotypic class is cumulated over all families. Family size is the offspring number of individual females. Transmission rate is the proportion of males carrying $\mathrm{fr}^{+}$among total number of males. Recombination rate is the proportion of recombinant phenotypes among males without $f r^{+}$.

All proportions and rates are calculated for each cross and given as median (and 1st quartiles) over all crosses.

$\dagger$ Proportion of males with pe allele (peach eye-colour phenotype) among males not harbouring $\mathrm{fr}^{+}$(expected proportion 0.5).

$\$$ Transmission rate of $\mathrm{fr}^{+}$to $s t+$ males (or recombinants pe + ) and to pepu males (or recombinants stpu) is the ratio of ' $++^{\prime}$ 'males

and of ' $+p u$ ' males to family size, respectively.

CF1 and were very similar at the $\mathrm{F}_{0}$ and $\mathrm{F}_{2}$ generations (0.73-0.82) (Table 1).

The genetic background had interesting effects on meiotic stability of CF2. The meiotic behaviour of CF2 was studied in $F_{1}$ and $F_{3}$ females in association with three different homologous chromosome genotypes. Overall, instability in meiosis was very high, with only $8 \%$ to $17 \%$ of the offspring inheriting the fragment (Table 2). However, a closer examination revealed a strong bias in segregation probabilities of $\mathrm{CF} 2$ with $p e$-vs. st-bearing chromosomes. First, the transmission rate through heterozygous $s t+/ p e p u f r^{+} \mathrm{F}_{1}$ females was decreased by a half relative to transmission through homozygous $s t+/ s t+f r^{+}$females (Table 2, MannWhitney test, $\left.U=167.5, n_{1}=49, n_{2}=61, P<0.001\right)$. Furthermore, the segregation pattern of CF2 in these heterozygous females was highly biased, such that very few purple ' $+p u$ ' males carrying the fragment were produced compared to wild-type ' ++ ' males (Table 2 ).

This unbalanced segregation of $\mathrm{CF} 2$ could be due to (a) preferential segregation of CF2 with $s t+$ chromosomes, (b) loss of the fragment when associated with рерu during meiotic segregation or (c) mortality of pepu $\mathrm{fr}^{+}$males. The data clearly show that CF2 did not preferentially segregate with $s t+$ in heterozygous females, because there was no increase in the frequency of $s t+f r^{+}$or decrease in $s t+$ offspring compared to the frequencies found from homozygous $s t+/ s t+f r^{+}$ females (Table 2). In addition, there was no significant decrease in family size of heterozygous vs. homozygous females (actually, the reverse is occurring: NewmanKeuls test, 108 d.f., $q=3.85$ ). In contrast, the data are consistent with the loss of CF2 chromosomes associated with pepu chromosomes during meiosis. The evidence is as follows. First, the reduction in frequency of phenotypic ' + pu' males (mostly pepu $\mathrm{fr}^{+}$but some stpu $\mathrm{fr}^{+}$ recombinant males) produced by heterozygous $s t+/$ pepu $\mathrm{fr}^{+}$females was balanced by a similar increase in the frequency of pepu males. In comparison to CF1 segregation ratios, the frequency of ' $+p u$ ' males declined from 0.093 to 0.004 (a decrease of 0.089), and was matched by an increase of 'st $p u$ ' males from 0.377 to 0.478 (an increase of 0.101). This result is best explained by the failure of CF2 to segregate with the рери chromosome during meiosis. The result is not consistent with the hypothesis that pepu $\mathrm{fr}^{+}$males are dying. Thus, we conclude that $\mathrm{CF} 2$ does not segregate stably with the pepu chromosome.

(C) The Genetics Society of Great Britain, Heredity, 87, 8-16. 

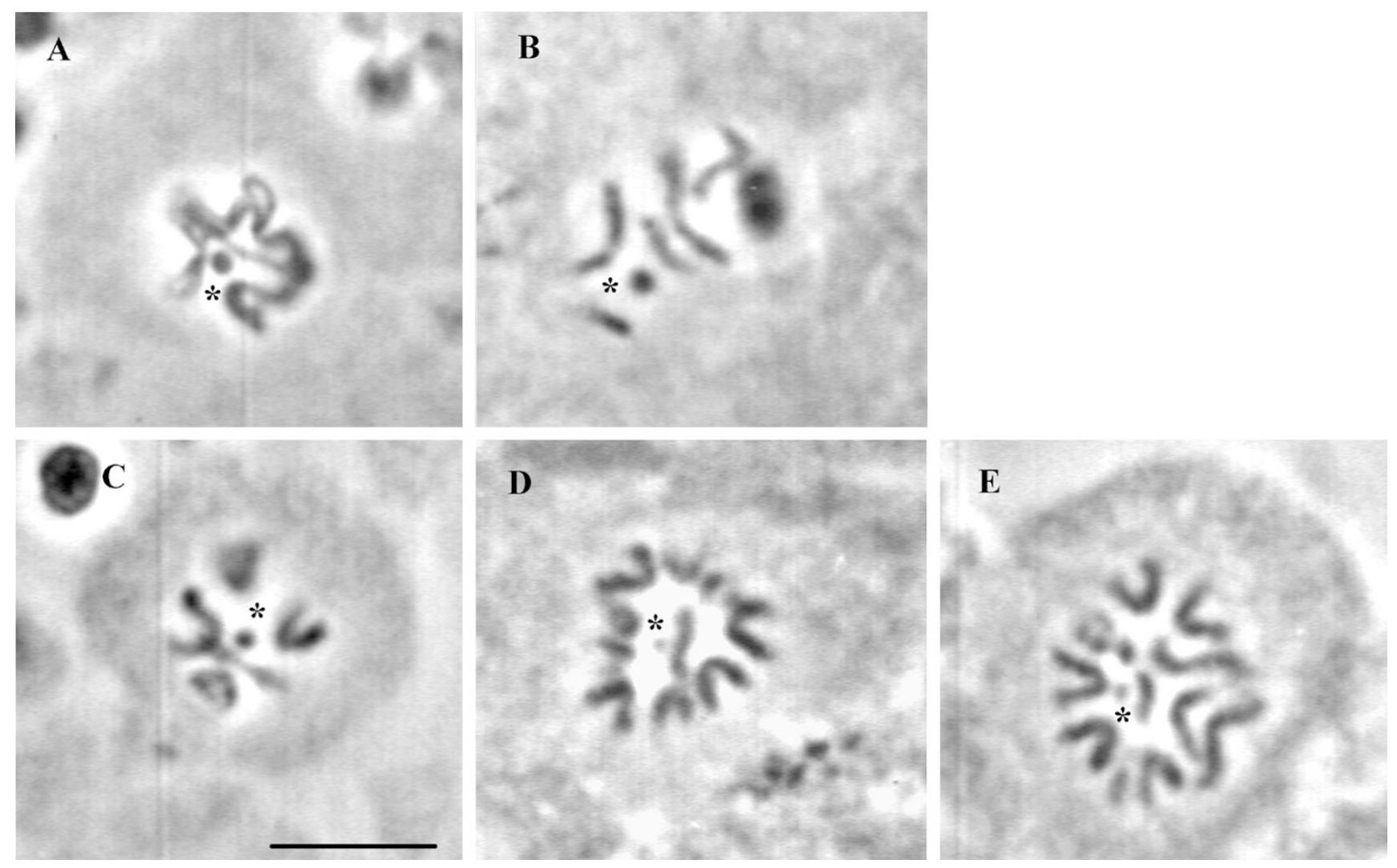

Fig. 1 A-E Polar views of metaphase brain cells from Nasonia. A, B, C: haploid male pupae $(n=5)$; E, F: diploid female pupae $(2 n=10)$. Note the central position of the centric fragment (asterisk) and its close association with an autosome in some cells. Bar, $10 \mu \mathrm{m}$.

To further investigate the CF2 transmission pattern in $s t+/$ pepu $\mathrm{fr}^{+}$females, we determined the genotype of 11 of their ' $+p u$ ' sons. Males were mated to $s t+/ s t+$ females. Four to six daughters carrying the fragment per $\mathrm{F}_{2}$ male were then mated to males from either St-DR, peach-purple, or Tinged ( $t i-277$; another $R$-complex eyecolour mutation) strains. Scoring of offspring phenotypes allowed us to determine the genotype of these $\mathrm{F}_{3}$ females and therefore of the $11^{\prime}+p u$ ' fathers. In 10 out of 11 cases, no peach offspring were produced from the $\mathrm{F}_{3}$ females when mated to pepu males (total number of offspring scored $n=1197$ ) nor from their sisters when mated to $s t+$ or $t i-277$ males (total number of male offspring scored $n=581$ ). Thus, we can confidently conclude that these $10^{\prime}+p u^{\prime} \quad \mathrm{F}_{2}$ males had the recombinant genotype stpu. In only one case did $\mathrm{F}_{3}$ sisters yield pepu male offspring, indicating that the genotype of their $\mathrm{F}_{2}$ ' + pu' father was pepu.

We therefore conclude that almost all the $27^{\prime}+p u$ ' found among $\mathrm{F}_{2}$ males (Table 2) were recombinants stpu $\mathrm{fr}^{+}$. The result is striking, given that the majority of ' $+p u$ ' males should have had the non-recombinant рерu genotype. The data strongly indicate that the region responsible for the instability of $\mathrm{CF} 2$ maps closely to the peach locus. From the recombination rate $(5 \%)$ and the CF2 transmission rate in meiosis $(17 \%)$, the expected frequency of recombinant chromosomes associated with $\mathrm{CF} 2$ among $\mathrm{F}_{2}$ males was $0.85 \%$, i.e. about 55 recombinants carrying $\mathrm{fr}^{+}$among the 6492 males produced (Table 2). There should then have also been approximately $28 \mathrm{pe}+\mathrm{fr}^{+}$recombinants among the ' ++ ' males. Although the genotype of ' ++ ' males has not been determined, it can be seen from Table 2 that, among recombinant males without $\mathrm{fr}^{+}$, there were 27 more pe + than stpu genotypes, although the increase is not significant (Chi squared, 141 vs. 166, $\chi^{2}=1.88,2$ d.f.). Nevertheless, the finding is consistent with the interpretation that CF2 was lost in most $\mathrm{pe}+\mathrm{fr}^{+}$males, as already observed in non-recombinant pepu $\mathrm{fr}^{+}$males. The instability of the fragment was therefore independent of the genotype at the bodycolour locus, but rather related to its association to the peach mutant at the $R$ locus in female meiosis.

The overall transmission rate of $\mathrm{CF} 2$ through the $\mathrm{F}_{3}$ heterozygous stpu/st $\mathrm{fr}^{+}$females (median and first quartiles $0.17(0.12-0.21), n=50)$ was not significantly

(C) The Genetics Society of Great Britain, Heredity, 87, 8-16. 
different from that for $\mathrm{F}_{1} s t+/ s t+f r^{+}$females, and significantly higher (about two-fold) than through $\mathrm{F}_{1}$ $s t+/$ pepu $\mathrm{fr}^{+}$females (Table 2, Mann-Whitney test, $\left.U=248.5, n_{1}=61, n_{2}=50, P<0.001\right)$. Moreover, no bias in the segregation of the fragment between stpu and $s t+$ chromosomes was observed among the progeny of these females when mated to pepu males, with $6 \%$ and $8 \%$ of the total progeny inheriting the fragment harbouring purple and wild-type body-colour, respectively. Therefore, CF2 was much more stable in association with stpu than with pepu chromosomes. Transmission rate through the four pepu/st $+f r^{+} \mathrm{F}_{3}$ females stemming from a single pepu $\mathrm{fr}^{+}$father was strongly decreased compared to transmission through $s t p u / s t+\mathrm{fr}^{+}$females (median and first quartiles, 0.065 (0.04-0.14) and $0.17(0.13-0.21)$, respectively; MannWhitney, $P<0.03)$. But it was not significantly decreased compared to $s t+/$ pepu $\mathrm{fr}^{+} \mathrm{F}_{1}$ females $(0.065$ (0.04-0.14) and $0.08 \quad(0.06-0.1)$, respectively). This observation further confirms the very high meiotic instability of the CF2 fragment with the peach mutant, independently of the parent from which the peach allele is inherited.

A curious effect of recombination between peach and purple was found on meiotic instability of CF2. Significant differences in $\mathrm{CF} 2$ transmission rate were observed among the stpu/st $+f r^{+} \mathrm{F}_{3}$ females of the 10 recombinant males (Kruskal-Wallis, $H=21.3,9$ d.f., $P=0.01)$. This paternal effect on meiotic instability could be due to genetic differences among $F_{2}$ males deriving individually from a unique recombination event in the heterozygous $F_{1}$ mother. More specifically, differences between recombinant chromosomes in pairing domains, resulting from recombination between the $R$-complex and $p u$ during meiosis, could differentially affect CF2 stability.

\section{Discussion}

Centric fragments are easy to generate in haplodiploid species, because aneuploid eggs can develop into viable and fertile haploid males, if few broken chromosomes of paternal origin are retained with the undamaged maternal haploid set. Mitotic-like spermatogenesis and meiotic oogenesis also allow a direct comparison of transmission rates between these two division processes in the same genetic background. We have shown here that (a) centric fragments (terminal deletions) can be generated using a chemical mutagen (EMS), (b) these chromosomes have reduced mitotic stability, presumably due to absence of telomeric sequences on their ends, and (c) the meiotic instability of such fragments can be influenced by the genetic structure of the homologous chromosomes.
Transmission pattern and instability of CF1 and CF2 are quite different, apart from their comparable transmission in homozygous $s t / s t$ female meiosis. Most important is the effect of genetic background on stability. The pattern of $\mathrm{CF} 2$ transmission observed over three generations revealed a strong meiotic instability of this fragment in association with the peach allele at the $R$ locus. This instability resulted in the loss of CF2 in meiosis of heterozygous $s t+/ p e p u$ females when associated either with pepu or with the recombinant $p e+$ chromosomes. Stability was restored in stpu recombinants, as shown in the transmission rate through heterozygous stpu/st $+f r^{+}$females. Thus, we can localize a zone of deficient meiotic interaction between the autosome and the fragment carrying the wild-type allele close to the pe allele in the $R$-complex.

The most plausible interpretation of the results presented here is that the peach-233 mutation is associated with a deletion (or rearrangement) that interferes with pairing and segregation of centric fragments lacking additional (distal) pairing domains, such as CF2. Consistent with this view is the observation that peach-233 appears to be a relatively large deletion, encompassing two complementation groups $(O+S)$ in the $R$ locus, whereas most $R$-locus mutations involve only one complementation group. According to one interpretation, $\mathrm{CF} 1$ is a larger centric fragment that contains additional pairing domains, maintaining its meiotic stability in association with the peach-233 mutation. These may represent specific pairing domains, or simply stretches of homology that affect stable segregation of the centric fragment in meiosis. Alternatively, CF2 may be longer, and regions of mismatch between it and the peach region could contribute to instability. Unfortunately, our cytological studies are insufficiently sensitive to distinguish size differences between the two fragments. Interestingly, presence of peach-233 in heterozygous $s t+/ p e p u$ females did not increase segregation of $\mathrm{CF} 2$ with the $s t+$ chromosome. This indicates that the initial pairing of the centric fragment with one or the other homologue in meiosis I is not a 'competitive' process, and is likely to be equal for the two homologues regardless of presence of the peach mutation. Loss of the centric fragment is more probably occurring during segregation of chromosomes at the first or second meiotic division, resulting in failure of $\mathrm{CF} 2$ to be incorporated into the ovum pronucleus. However, it is clear that more detailed cytological studies are needed to test this hypothesis.

Differences in mitotic instability of the two centric fragments were also present, with CF2 showing higher mitotic stability (0.73-0.82 transmission through males) than CF1 (0.38 transmission through males). This pattern could be due to differences in the size of these

(c) The Genetics Society of Great Britain, Heredity, 87, 8-16. 
fragments, with the (presumably) larger CF1 fragment showing reduced mitotic stability. This hypothesis would run against the size-related stability reported for B chromosomes: large Bs are usually mitotically more stable than small ones (Hewitt, 1979). However, several important structural differences between 'young' centric fragments and evolved B chromosomes may account for such a discrepancy, such as telomeric acquisition and heterochromatization. These processes are suspected or known to occur during B-chromosome evolution and to contribute to their synaptic isolation and independent meiotic/mitotic behaviour (Camacho et al., 2000). For future analysis, molecular markers are now available (Gadau et al., 1999; Opijnen \& Werren, unpublished) to more accurately assess size of centric fragments.

Our results may be relevant to questions concerning the origin and evolution of B-chromosomes. B-chromosomes are extra (or supernumerary) chromosomes found in many animal and plant species (Jones, 1985; Werren et al., 1988; Shaw \& Hewitt, 1990; Camacho et al., 2000). A standing hypothesis on the evolution of B-chromosomes is their origin as chromosomal fragments, either produced as duplication of normal autosomes or by chromosome fragmentation in interspecific hybridization (Werren et al., 1988; Shaw \& Hewitt, 1990; Camacho et al., 2000; Perfectti \& Werren, 2001). The study of chromosomal fragment stability may thus contribute to our understanding of early steps in the evolution of B-chromosomes, in particular their accumulation pattern. An extreme example of a 'parasitic' B-chromosome is the Paternal Sex Ratio (PSR) found in N. vitripennis (Nur et al., 1988; Werren, 1991; Beukeboom \& Werren, 1993a). Terminal deletions have been generated in the PSR chromosome as a tool for genetic analysis. These studies show that transmission rate of nonfunctional deletion PSR chromosomes were generally high (around 75\%) through males and low (around $10 \%$ ) through females (Beukeboom \& Werren, 1993b). Between-male variability in transmission rates was also reported, both for nonfunctional deletion PSR (around $75 \%$ ), regular PSR (from 94 to $100 \%$ ) chromosomes, and deletion PSR chromosomes (Beukeboom \& Werren, 1993b). Thus, it can be concluded that terminal deletions tended to increase the instability of the PSR chromosome. Such instability could be due to deletions of telomeric terminal regions (Beukeboom \& Werren, 1993b), accounting for occasional mitotic loss.

Evidence suggests that PSR was introduced into $N$. vitripennis via an interspecific hybridization, possibly also initially as a centric fragment (Reed, 1993; McAllister \& Werren, 1997), with subsequent amplification of unique repetitive DNA sequences (Eickbush et al., 1992). A neo-B chromosome has recently been recovered in hybrids of $N$. vitripennis and $N$. giraulti showing a pattern of meiotic and mitotic instability comparable to CF2 (Perfectti \& Werren, 2001). Due to reduced mitotic and meiotic transmission, a neo-PSR chromosome must quickly acquire its ability to cause paternal genome loss in fertilized eggs (thus increasing its presence in males, the sex with higher transmission), or the centric fragment will be rapidly lost. The same is true for other B-chromosomes that were generated initially as centric fragments; accumulation mechanisms must quickly arise to counteract the reduced transmission likely to occur subsequent to their origin. In particular, telomeric healing of broken ends would be needed to avoid degradation and shortening of the CF (Zakian, 1995), thereby increasing its mitotic and meiotic stability partly by protecting pairing domains. In the two centric fragments studied here $(\mathrm{CF} 1$ and $\mathrm{CF} 2$ ), it is likely that no telomeric sequences were present, and their transmission rates were strikingly similar to those of nonfunctional deletion PSR having lost telomeric terminal regions, and of a neo-B of interspecific origin (Perfectti \& Werren, 2001). An increase in the transmission rate and mitotic stability of the latter was observed over successive generations, reaching nearly $100 \%$ (Perfectti \& Werren, 2001). We have indirect genetic evidence (increased mitotic stability over successive generations) of telomeric healing of terminal deletions in Nasonia (McAllister \& Werren, unpublished). However, the actual telomeric sequence of Nasonia is not yet known. Once this is determined, the Nasonia system could be a useful one for studying telomeric acquisition at the molecular genetic level, given the relative ease of generating and maintaining terminal deletions in this species.

\section{Acknowledgements}

Support for this research was provided by the U.S. National Science Foundation (J. H. W.), and a postdoctoral Lavoisier grant from the French Ministry of Foreign Affairs to M.-J. P.-M.

\section{References}

AHMAD, K. AND GOLIC, K. G. 1998. The transmission of fragmented chromosomes in Drosophila melanogaster. Genetics, 148, 775-792.

BEUKEBOOM, L. W. AND WERREN, J. H. 1993a. Transmission and expression of the parasitic paternal sex ratio (PSR) chromosome. Heredity, 70, 437-443.

BEUKeboom, L. W. AND WERREN, J. H. 1993b. Deletion analysis of the selfish B chromosome, Paternal Sex Ratio (PSR), in the parasitic wasp Nasonia vitripennis. Genetics, 133, 637-648.

CAMACHO, J. P. M., SHARBEL, T. F. AND BEUKEBOOM, L. W. 2000. B-chromosome evolution. Phil. Trans. R. Soc. B, 355, 163-178. 
EICKBUSH, D., EICKBUSH, T. AND WERREN, J. H. 1992. Molecular characterization of repetitive DNA sequences from a B chromosome. Chromosoma, 101, 575-583.

GADAU, J., PAGE, R. AND WERREN, J. H. 1999. Mapping of hybrid incompatibility loci in Nasonia. Genetics, 153, 1731-1741.

GRANT, W. F. AND OWENS, E. T. 1998. Chromosome aberration assays in Crepis for the study of environmental mutagens. Mutat. Res., 410, 291-307.

GRigliatti, T. 1986. Mutagenesis. In: Roberts, D. (ed.) Drosophila: A Practical Approach, pp. 39-52. IRL Press, Washington DC.

HEwITT, G. M. 1979. Animal Cytogenetics, 3: Insecta 1: Orthoptera: Grasshoppers and Crickets. Gebrüder Borntraeger, Berlin.

JONES, R. N. 1985. Are B chromosomes 'selfish'?. In: CavalierSmith, T. (ed). The Evolution of Genome Size, pp. 397-425. John Wiley \& Sons, New York.

LI, M. M., HOWARD-PEEBLES, P. N., KILLOS, L. D., FALLON, L. ET $A L$. 2000. Characterization and clinical implications of marker chromosomes identified at prenatal diagnosis. Prenatal Diagnosis, 20, 138-143.

MCALLISTER, B. F. AND WERREN, J. H. 1997. Hybrid origin of a B chromosome (PSR) in the parasitic wasp Nasonia vitripennis. Chromosoma, 106, 243-253.

MCCLINTOCK, B. 1942. The fusion of broken ends of chromosomes following nuclear fusion. Genetics, 28, 458-463.

NUR, U., WERren, J. H., EICKBUSH, D. G., BURKE, W. D. ET AL. 1988. A 'selfish' B chromosome that enhances its transmission by eliminating the paternal chromosomes. Science, $\mathbf{2 4 0}$, $512-514$.

PAI, S. A., CHEUNG, M. C., ROMSDAHL, M. M., MULTANI, A. S. ET $A L$. 1999. Can genetic instability be studied at the single chromosome level in cancer cells? Evidence from human melanoma cells. Cancer Genet. Cytogenet., 109, 51-57.

PERFECTTI, F. AND WERREN, J. H. 2001. The interspecific origin of B chromosomes: experimental evidence. Evolution, 55, 1069-1073.
REED, K. 1993. Cytogenetic analysis of the paternal sex ratio chromosome of Nasonia vitripennis. Genome, 36, 157-161.

RYAN, S. L., SAUL, G. B. AND CONNER, G. W. 1985. Aberrant segregation of $R$-locus genes in male progeny from incompatible crosses in Mormoniella. J. Hered., 76, 21-26.

RYAN, S. L., SAUL, G. B. AND CONNER, G. W. 1987. Separation of factors containing $R$-locus genes in Mormoniella stocks derived from aberrant segregation following incompatible crosses. J. Hered., 78, 273-275.

SANKARANARAYANAN, K. 1996. Environmental chemical mutagens and genetic risks: lessons from radiation genetics. Environ. Mol. Mutagen., 28, 65-70.

SAUL, G. B. II. 1989. Gene map of the parasitic wasp Nasonia vitripennis ( $=$ Mormoniella vitripennis). In: O'Brien, S. J. (ed.) Genetic Maps: Locus Maps of Complex Genomes, pp. 198-201. Cold Spring Harbor Laboratory Press, Cold Spring Harbor, NY.

SAUL, G. B. II., Whiting, P. W., SAUl, S. W. AND HEIDNER, C. A. 1965. Wild-type and mutant stocks of Mormoniella. Genetics, 52, 1317-1327.

SHAW, M. W. AND HEWITT, G. M. 1990. B chromosomes, selfish DNA and theoretical models: where next?. In: Futuyma, D. and Antonovics, J. (eds) Oxford Surveys in Evolutionary Biology, vol. 7, pp. 197-223. Oxford University Press, New York.

STANKIEWICZ, P., BOCIAN, E., JAKUBOW-DURSKA, K., OBERSZTYN, E. ET AL. 2000. Identification of supernumerary marker chromosomes derived from chromosomes 5, 6, 19, and 20 using FISH. J. Med. Genet., 37, 114-120.

WERREN, J. H. 1991. The PSR (paternal sex ratio) chromosome. Amer. Nat., 137, 392-402.

WERREN, J. H., NUR, U. AND WU, C. I. 1988. Selfish genetic elements. Trends Ecol. Evol., 11, 297-302.

ZAKIAN, v. A. 1995. Telomeres: beginning to understand the end. Science, 270, 1601-1606. 